\title{
Respiratory Volume Monitoring to Assess the Effect of Airway Maneuvers on Ventilation during Upper Endoscopy
}

\author{
Katherine Holley ${ }^{1}$, Donald Mathews ${ }^{1}$, Diane Ladd ${ }^{2,3 *}$, Lisa Campana ${ }^{3}$, Howard Schapiro ${ }^{1}$ \\ ${ }^{1}$ Department of Anesthesiology, Fletcher Allen Health Care, University of Vermont College of Medicine, \\ Burlington, USA \\ ${ }^{2}$ School of Nursing, West Virginia University, Morgantown, USA \\ ${ }^{3}$ Respiratory Motion, Inc., Waltham, USA \\ Email: dladd@respiratorymotion.com, ${ }^{*}$ dladd@hsc.wvu.edu
}

Received 10 September 2014; revised 15 October 2014; accepted 6 November 2014

Academic Editor: Nazish Khalid Hashmi, Duke University Hospital, USA

Copyright (C) 2014 by authors and Scientific Research Publishing Inc.

This work is licensed under the Creative Commons Attribution International License (CC BY).

http://creativecommons.org/licenses/by/4.0/

(c) (7) Open Access

\begin{abstract}
Introduction: Propofol use during endoscopic procedures has become increasingly popular and assessing and maintaining airway patency is a significant challenge. Anesthesiologists often use airway maneuvers to maintain airway patency and ventilation during procedural sedation. A novel, non-invasive, Respiratory Volume Monitor (RVM) that provided continuous, real-time measurements of minute ventilation (MV), tidal volume (TV) and respiratory rate (RR) was used to monitor respiratory performance before, during, and after endoscopic procedures, quantify MV changes before and after airway maneuvers, and to quantify propofol-induced respiratory depression. Methods: RVM traces were obtained from 25 patients undergoing sedation for endoscopic procedures. Airway maneuvers were performed in 19/25 patients. All 25 patients received propofol as the primary sedative. Results: Forty-five airway maneuvers were performed. During these maneuvers, all respiratory parameters increased relative to pre-maneuver levels. On average, MV increased by $24 \% \pm 5 \%$ (mean \pm SEM), TV $14 \% \pm 5 \%$ and RR: $17 \% \pm 6 \%$. The cohort average $\mathrm{MV}_{\text {BASELINE }}$ was $9.5 \pm 0.7 \mathrm{~L} / \mathrm{min}(\mathrm{TV}=670 \pm 60 \mathrm{ml}, \mathrm{RR}=15 \pm 0.7$ ). Following propofol MV decreased transiently, reaching nadir five minutes after the last dose of propofol at $82 \% \pm 10 \%$ of baseline (MV = 7.5 $\pm 1.0 \mathrm{~L} / \mathrm{min})$. The reduction in MV was driven by reduction in TV, not RR. Conclusions: Data demonstrated that RVM was able to track changes in ventilation and was able to quantify respiratory changes following airway maneuvers. All patients had a significant reduction in ventilatory volumes after propofol. Five minutes after the last dose of propofol, MV and TV were signif-
\end{abstract}

\footnotetext{
"Corresponding author.
}

How to cite this paper: Holley, K., Mathews, D., Ladd, D., Campana, L. and Schapiro, H. (2014) Respiratory Volume Monitoring to Assess the Effect of Airway Maneuvers on Ventilation during Upper Endoscopy. Open Journal of Anesthesiology, 4, 281-290. http://dx.doi.org/10.4236/ojanes.2014.411041 
icantly reduced while RR was not, suggesting that monitoring respiratory rate alone was not a sufficient indicator of respiratory status.

\title{
Keywords
}

\author{
Non-Invasive Respiratory Volume Monitoring, Minute Ventilation, Airway Maneuvers, Procedural \\ Sedation, Endoscopy
}

\section{Introduction}

Over 20 million gastrointestinal endoscopies are performed each year in the United States [1]. Most of these procedures required conscious sedation achieved through a combination of benzodiazepines, opiates and other anesthetic agents [2]. Deeper levels of sedation may be necessary for certain procedures such as endoscopic ultrasound (EUS) and endoscopic retrograde cholangiopancreatography (ERCP) [2]. The use of propofol is becoming increasingly popular to achieve rapid and deeper levels of sedation during endoscopic procedures [3] [4]. Propofol has a short half-life (2 - 4 minutes), leading to a decrease in post-procedural recovery times [3]. However, propofol-induced deep sedation may increase the incidence of respiratory depression and apnea [5]-[8] increasing the need for continuous airway and ventilation monitoring.

Assessing and maintaining airway patency under propofol sedation can be a significant challenge. Propofol decrease respiratory drive in a dose-dependent fashion, but propofol also causes loss of muscle tone in the muscles of the upper airway, which may result in partial or complete airway collapse and ultimately may lead to respiratory decompensation. Monitoring by trained anesthesiology providers is beneficial, but an objective, quantitative measurement may be helpful in difficult patients and useful for less experienced providers. Further, troubleshooting the cause of airway obstruction is especially difficult during endoscopic procedures where patient positioning can create access problems and a portion of the airway is compromised by the endoscope.

Anesthesiologists often rely on common airway maneuvers, such as the chin lift and jaw thrust, to maintain a patent airway and ensure adequate ventilation during procedural sedation. Unfortunately, it can be difficult to determine when such a maneuver is necessary and to properly monitor and quantify the effectiveness of a maneuver in real time. Monitoring with pulse oximetry is often inadequate due to intermittent dislodgement and the inherent delay between reductions in ventilation and a drop in blood oxygen levels [9]. Propofol-induced respiratory depressions and apnea may persist unmonitored during the recovery period post-procedure, leading to potential complications.

A novel, non-invasive, Respiratory Volume Monitor (RVM) has been shown to provide accurate, continuous, real-time measurements of minute ventilation (MV), tidal volume (TV) and respiratory rate (RR) in a variety of ambulatory patients [10]. This study was designed to demonstrate that the RVM could adequately monitor respiratory performance before, during, and after endoscopy procedures, specifically addressing whether RVM can: 1) quantify the change in MV from before and after an airway maneuver and 2) quantify respiratory depression induced by commonly-used sedatives.

\section{Methods}

\subsection{Patients}

Patients undergoing sedation for upper endoscopic procedures, including endoscopic ultrasound (EUS), esophagogastroduodenoscopy (EGD), gastroscopy, and endoscopic retrograde cholangiopancreatography (ERCP) were eligible for enrollment. Exclusion criteria included: history of thoracotomy with resection of lung tissue, severe chronic obstructive pulmonary disease (defined as an inability to climb a flight of stairs) or forced expiratory volume in one-minute divided by vital capacity (FEV1/VC) of less than $30 \%$ of predicted), or a body mass index (BMI) of greater than $50 \mathrm{~kg} / \mathrm{m}^{2}$. The protocol was approved by the Fletcher Allen Health Care Institutional Review Board and all patients gave written informed consent prior to enrollment.

\subsection{Instrumentation}

A bio-impedance based RVM (ExSpiron, Respiratory Motion, Inc., Waltham MA) was used to collect digital 
respiratory traces from an electrode PadSet placed on the thorax from 25 patients (mean age: 54.1, range: 20 - 83 years; mean BMI: 27.6, range: $21-46 \mathrm{~kg} / \mathrm{m}^{2}$ ) undergoing upper endoscopic procedures. Healthcare providers were blinded to RVM data; however a unit-less respiratory curve was displayed to ensure device function. The RVM PadSet electrodes were placed at the sternal notch, xiphoid and right mid-axillary line at the level of the xiphoid [10]. With recommended electrode placement (Figure 1) and advanced algorithms strong correlations (0.96 \pm 0.16 , mean $\pm 95 \%$ CI for regular and erratic breathing) and high accuracy (average MV and TV errors less than $10 \%$ and average RR error less than $2 \%$ ) between RVM and spirometric measurements have been previously demonstrated [10].

\subsection{Procedure}

After obtaining written informed consent, each subject performed 2 - 4 spirometer tests. For each spirometry test, the patient was asked to breathe through a Wright/Haloscale Respirometer (nSpire Health, Inc., Longmont, CO, "Wright") for 60 seconds. The minute ventilation measured by the Wright spirometer was entered into the RVM. The spirometry measurements were performed in both the supine and left lateral decubitus positions. The number of tests performed varied with the patient's ability and the available time before the procedure. RVM traces were recorded continuously from pre-procedure until discharge. During the procedure end-tidal $\mathrm{CO}_{2}$ (Philips Smart CapnoLine Adult $\mathrm{CO}_{2} / \mathrm{O}_{2}$ nasal cannula), and pulse oximetry were measured. The anesthesia care team cared for the patients as per the usual standard of care.

Twenty-five patients were evaluated. Procedures the patients underwent included: 15 EGD (1 with colonoscopy, 1 with ERCP, 1 with manometry), 4 ERCP, 4 EUS, and 2 gastroscopies. MV, TV, and RR were calculated from 30 -second segments over the patient's entire stay from pre-procedure through discharge. Average MV, TV, and RR were calculated from a two-minute period of stable breathing prior to the procedure, this was characterized as the patient's baseline. Predicted MV for each patient was calculated based on the estimated body surface area [11] (BSA) (Equations (1)-(3)).

$$
\begin{gathered}
\text { BSA }=0.0101 *\left(\text { Weight }^{0.425}\right) *\left(\text { Height }^{0.725}\right) \\
\text { Females }[12]: \text { MV }=\text { BSA } * 3.5 \\
\text { Males }[12]: \text { MV }=\text { BSA } * 4.0
\end{gathered}
$$

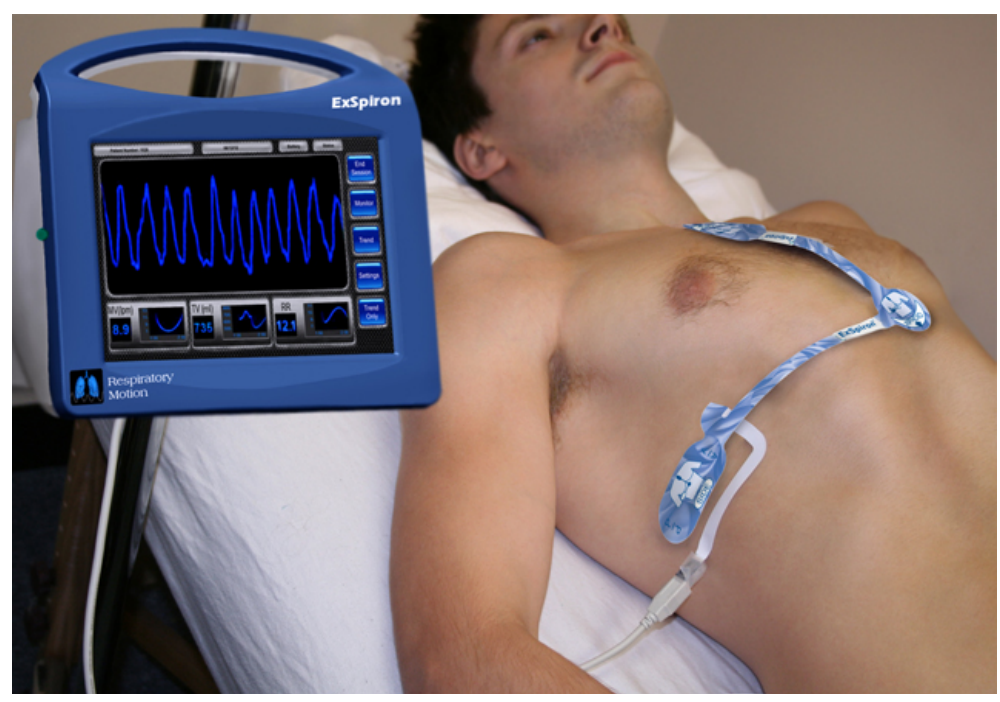

Figure 1. PadSet placement: A non-invasive Respiratory Volume Monitor (RVM, ExSpiron, Respiratory Motion, Inc.) that provides continuous, realtime, non-invasive measurements of minute ventilation (MV), tidal volume (TV) and respiratory rate (RR). Figure shows standard electrode placement. The PadSet electrode pads are placed on the sternal notch and xiphoid. The third pad is placed along the right mid-axillary line at the level of the xiphoid. 


\subsection{Airway Maneuvers}

Airway maneuvers evaluated included chin lifts and jaw thrusts. If two maneuvers occurred within one minute of one another, the second maneuver was discarded from the analysis. Additionally, if a maneuver occurred during the insertion or removal of the endoscope, the maneuver was discarded from the analysis. Average MV, $\mathrm{TV}$, and RR were calculated one minute before each airway maneuver and then again during the maneuver at the peak ventilation.

\subsection{Medications}

The timing and dosage of propofol and other sedatives were recorded. Propofol delivery was based on the administering anesthesiologist's preference and was given either by continuous infusion or as multiple IV boluses. In order to homogenize the dataset across patients and modes of delivery, the effects of propofol on ventilation were analyzed during the time period around the last bolus of propofol or at the end of the infusion, which always occurred near the end of the procedure. Two-minute averages of MV, TV, and RR were calculated five minutes before the last dose of propofol and every five minutes after the last dose of propofol for 30 minutes. All 25 patients were included in this analysis.

\subsection{Data Analyses}

For each analysis, MV, TV, and RR were evaluated both as absolute values and as percentages of baseline ventilation. The average population estimates of these values are presented in this manuscript as mean \pm standard error of the mean (SEM). Airway maneuvers were analyzed separately according to type of maneuver: jaw thrust or chin lift. All jaw thrust maneuvers were pooled and average MV, TV, and RR were calculated pre- and post-maneuver. Similarly, all chin lift maneuvers were pooled and average MV, TV, and RR was calculated preand post-maneuver. Paired t-tests were used to reject the null hypothesis that a maneuver resulted in no-change in ventilation (post-maneuver values were compared to pre-maneuver values).

To determine the effect of propofol on ventilation, average MV, TV, and RR were calculated across the subject population at baseline and at time points near the last dose of propofol. To assess differences in ventilation from baseline to pre- and post-propofol dose, a repeated measures single-factor analysis of variance (ANOVA) was used. For the primary ANOVA, the null hypothesis was that there would be no change in ventilation before or after propofol as compared to baseline. A follow up post-hoc analysis was conducted to determine which time points were significantly different from baseline. To assess absolute differences in MV, TV, and RR, paired t-tests were used before and after propofol administration as compared to baseline. To assess relative differences in MV, TV, and RR, a one-sided t-test was used to determine if the percent change in MV, TV, and RR from baseline was significantly different from zero. The distributions of MV, TV, and RR measurements were tested for normality across the patient cohort.

\section{Results}

\subsection{Subject Demographics}

Patient demographics, procedure types, and medications given are summarized in Table 1. Airway maneuvers were performed in 19 of the 25 non-ventilated patients. All 25 patients received propofol as the primary sedative and most received one or more additional medications such as ketamine (15 patients), fentanyl (3 patients), or midazolam (17 patients).

\subsection{Airway Maneuvers}

A total of 45 airway maneuvers were performed in 19 patients. Of those 45 maneuvers, 15 occurred during the insertion or removal of the endoscope (11 chin lifts, 3 jaw thrusts, and 1 chin-lift-jaw-thrust combination) and seven occurred immediately following another maneuver and were excluded from this analysis. Of the 20 maneuvers analyzed here, 9 were chin lifts, 11 were jaw thrust. In this cohort, the monitored respiratory parameters increased following an airway maneuver relative to pre-maneuver levels. MV increased on average by $24 \% \pm$ $5 \%$ (mean \pm SEM), TV increased by $14 \% \pm 5 \%$ and RR increased by $17 \% \pm 6 \%$.

Figure 2(a) shows an example of a jaw thrust in an 80-year-old female patient undergoing EUS. Predicted 

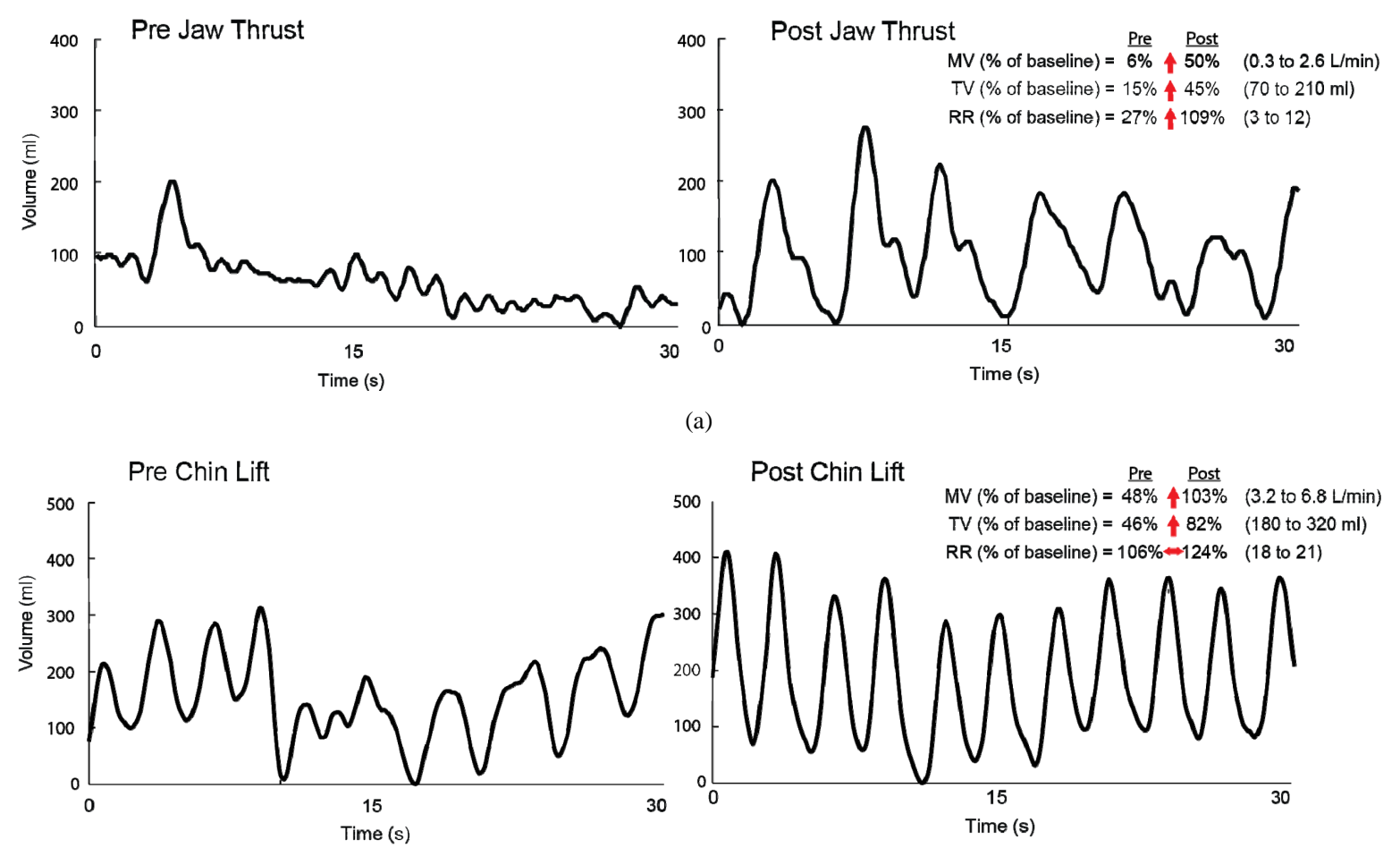

(a)

(b)

Figure 2. Examples of the effectiveness of Airway Maneuvers: (a) Example Respiratory Volume Monitor (RVM) respiratory traces and calculated respiratory volume and rate measurements from an esophagogastroduodenoscopy (EGD) patient before and after a jaw thrust performed by an anesthesiologist to improve airflow (blinded to RVM data). At baseline, prior to any sedative administration, the baseline minute ventilation ( $\mathrm{MV}_{\text {BASELINE }}$ ) was $5.2 \mathrm{~L} / \mathrm{min}$, close to the predicted minute ventilation ( $\mathrm{MV}_{\mathrm{PRED}}$ ) value of $4.9 \mathrm{~L} / \mathrm{min}$. Before the maneuver, the minute ventilation (MV) and tidal volume (TV) had decreased. The $\mathrm{MV}$ to $6 \%$ of $\mathrm{MV}_{\mathrm{BASELINE}}(\mathrm{MV}=0.3 \mathrm{~L} / \mathrm{min}, \mathrm{TV}=70 \mathrm{ml}, \mathrm{RR}=3 \mathrm{breaths} / \mathrm{min}$, left panel). After the maneuver, the ventilation improved significantly, but was still only $50 \%$ of $\mathrm{MV}_{\text {BASELINE }}(\mathrm{MV}=2.6 \mathrm{~L} / \mathrm{min}, \mathrm{TV}=210 \mathrm{ml}, \mathrm{RR}=12 \mathrm{breaths} / \mathrm{min}$, right panel); (b) Example RVM respiratory traces and calculated respiratory volume and rate measurements from an EGD patient before and after a chin lift performed by an anesthesiologist to improve airflow (blinded to RVM data). MV $\mathrm{BASELINE}_{\text {, }}$ prior to any sedative administration, is $6.6 \mathrm{~L} / \mathrm{min}$, above the $\mathrm{MV}_{\text {PREDICTED }}=5.6 \mathrm{~L} / \mathrm{min}$. Before the maneuver, $\mathrm{MV}$ had decreased to $48 \%$ of $\mathrm{MV}_{\text {BASELINE }}(\mathrm{MV}=3.2 \mathrm{~L} / \mathrm{min}$, $\mathrm{TV}=180 \mathrm{ml}, \mathrm{RR}=18 \mathrm{breaths} / \mathrm{min}$, left panel). After the maneuver the ventilation doubled to $103 \%$ of $\mathrm{MV}_{\text {BASELINE }}$ (right panel). Note that the $\mathrm{MV}$ increased to $6.8 \mathrm{~L} / \mathrm{min}$ was due to a larger increase in tidal volume (TV) (from 180 to $320 \mathrm{ml}$ ) than an increase in RR (from 18 to 21 breaths/min).

Table 1. Patient demographics, predicted minute ventilation $\left(\mathrm{MV}_{\mathrm{PRED}}\right)$, procedure type, medication administration mode and dosage for the cohort.

\begin{tabular}{|c|c|c|c|c|c|c|c|}
\hline Procedure & Gender & $\begin{array}{l}\text { Age } \\
\text { (Years) }\end{array}$ & $\begin{array}{l}\text { Body Mass Index } \\
(\mathrm{BMI})\left(\mathrm{kg} / \mathrm{m}^{2}\right)\end{array}$ & $\begin{array}{c}\text { Predicted Minute } \\
\text { Ventilation (MV } \text { PRED })\end{array}$ & $\begin{array}{l}\text { Procedure Length } \\
\text { (min) }\end{array}$ & $\begin{array}{c}\text { Propofol Delivery } \\
\text { Method (Infusion/Bolus) }\end{array}$ & $\begin{array}{l}\text { Propofol Dose } \\
\text { (mg) }\end{array}$ \\
\hline $\begin{array}{c}\text { EDG } \\
(\mathrm{n}=12)\end{array}$ & $8 \mathrm{~F}, 4 \mathrm{M}$ & $50.1 \pm 7.9$ & $28.7 \pm 7.6$ & $6.9 \pm 1.0$ & $4.3 \pm 2.5$ & $\begin{array}{l}\text { Infusion }=7 \\
\text { Bolus }=5\end{array}$ & $141 \pm 61.7$ \\
\hline $\begin{array}{l}\text { ERCP } \\
(\mathrm{n}=4)\end{array}$ & $2 \mathrm{~F}, 2 \mathrm{M}$ & $69.8 \pm 11.4$ & $26.0 \pm 5.2$ & $7.6 \pm 1.4$ & $28.5 \pm 6.8$ & Infusion $=4$ & $270.0 \pm 56.3$ \\
\hline $\begin{array}{l}\text { EUS } \\
(n=4)\end{array}$ & $2 \mathrm{~F}, 2 \mathrm{M}$ & $55.0 \pm 17.5$ & $24.3 \pm 3.6$ & $6.4 \pm 1.4$ & $28.3 \pm 7.3$ & $\begin{array}{c}\text { Infusion }=1 \\
\text { Bolus }=3\end{array}$ & $320.0 \pm 165.1$ \\
\hline $\begin{array}{l}\text { Other } \\
(\mathrm{n}=5)\end{array}$ & $2 \mathrm{~F}, 3 \mathrm{M}$ & $50.6 \pm 23.3$ & $29.0 \pm 1.9$ & $7.1 \pm 1.0$ & $15.8 \pm 11.8$ & $\begin{array}{l}\text { Infusion }=2 \\
\text { Bolus }=3\end{array}$ & $266.2 \pm 110.6$ \\
\hline
\end{tabular}

EGD: esophagogastroduodenoscopy, ERCP: endoscopic retrograde cholangiopancreatography, EUS: endoscopic ultrasound, Other: combination studies and gastroscopies.

MV ( $\mathrm{MV}_{\text {PRED }}$, based on body surface area) for this patient was $4.9 \mathrm{~L} / \mathrm{min}$ and the Baseline MV (MV $\mathrm{BASELINE}_{\text {), }}$ recorded after placing the patient in the left lateral position for the procedure and prior to the administration of 
any sedatives or other anesthetics, was $5.2 \mathrm{~L} / \mathrm{min}\left(106 \%\right.$ of $\left.\mathrm{MV}_{\mathrm{PRED}}\right)$. During the procedure and prior to the anesthesiologist initiating the jaw thrust maneuver, the patient exhibited a 25-second-long period of apnea, resulting in a MV measured by the RVM monitor of $6 \%$ of $\mathrm{MV}_{\text {BASELINE }}(\mathrm{MV}=0.3 \mathrm{~L} / \mathrm{min}, \mathrm{TV}=70 \mathrm{ml}, \mathrm{RR}=3$ ), shown in the left panel of Figure 2(a). This apnea likely prompted the anesthesiologist to perform a jaw thrust. Following the maneuver, airway patency was partially restored, as demonstrated by an 8-fold increase in MV to $50 \%$ of $\mathrm{MV}_{\text {BASELINE }}(\mathrm{MV}=2.6 \mathrm{~L} / \mathrm{min}, \mathrm{TV}=210 \mathrm{ml}, \mathrm{RR}=12)$, as shown in the right panel of Figure 2(a). While this airway maneuver was implemented based on the clinical observation of obstructive apnea and led to a clear improvement in ventilation, the resulting MV remained at half of baseline, a deficiency that could not be observed without the advanced real-time monitoring capabilities of the RVM.

Figure 2(b) shows an example of an effective chin lift in a 41-year-old female also undergoing EUS with an $\mathrm{MV}_{\text {PRED }}$ of $5.6 \mathrm{~L} / \mathrm{min}$ and a measured $\mathrm{MV}_{\text {BASELINE }}$ of $6.6 \mathrm{~L} / \mathrm{min}$. The baseline $\mathrm{MV}$ in this patient was slightly above $\mathrm{MV}_{\mathrm{PRED}}$, possibly due to pre-procedural anxiety. Prior to the chin-lift the RVM recorded MV at $48 \%$ of $\mathrm{MV}_{\text {BASELINE}}$, secondary to shallow breathing $(\mathrm{MV}=3.2, \mathrm{TV}=180 \mathrm{ml}, \mathrm{RR}=18)$, as shown in the left panel of Figure 2(b). After the maneuver, the measured MV more than doubled to $103 \%$ of $\mathrm{MV}_{\text {BASELINE }}$ (MV $=6.8$ $\mathrm{L} / \mathrm{min}, \mathrm{TV}=320 \mathrm{ml}, \mathrm{RR}=21$ ), confirming the efficacy of the maneuver (right panel of Figure 2(b)).

The effectiveness of airway maneuvers was evident across all patients studied here, as both jaw thrusts and chin lifts improved airway patency (Figure 3). Jaw thrusts increased MV, on average, from $4.1 \pm 0.7 \mathrm{~L} / \mathrm{min}$ to $6.1 \pm 1.0 \mathrm{~L} / \mathrm{min}(25 \% \pm 6 \%$, range: $-12 \%$ to $60 \%)$ and chin lifts increased MV from $5.0 \pm 1.0 \mathrm{~L} / \mathrm{min}$ to $6.8 \pm 1.1$ $\mathrm{L} / \mathrm{min}(21 \% \pm 9 \%$, range: $-12 \%$ to $62 \%)$. Both improvements were significant (paired t-tests, ${ }^{*} \mathrm{p}<0.01$ and ${ }^{\mathrm{F}} \mathrm{p}<$ 0.05 respectively).

While it is known that airway maneuvers can improve ventilation and help with airway management, there is currently no measure of adequacy of ventilation in non-intubated patients or any way to provide quantitative feedback of the effectiveness of the maneuver in restoring ventilation. In this study, the RVM recorded several instances of airway compromise that remained unnoticed by the clinical staff. Figure 4 shows an example of unnoticed and potentially dangerous respiratory compromise in a 20-year-old female undergoing an EGD/ERCP. Prior to any sedatives being given $\mathrm{MV}_{\text {BASELINE }}$ was $7.3 \mathrm{~L} / \mathrm{min}, 16 \%$ above the predicted value for this patient of $6.3 \mathrm{~L} / \mathrm{min}$. To initiate sedation prior to procedure start, the patient was given $2 \mathrm{mg}$ midazolam, $20 \mathrm{mg}$ ketamine, and $100 \mathrm{mg}$ propofol in two boluses. Soon after the administration of these sedatives and near the beginning of the procedure (Figure 4, left), MV was measured at $82 \%$ of baseline ( $\mathrm{MV}=6.0 \mathrm{~L} / \mathrm{min}$, TV $=300 \mathrm{ml}, \mathrm{RR}=20$ ). However, 3 minutes later, the patient received another bolus of $50 \mathrm{mg}$ propofol and the RVM recorded a substantial drop in MV to $16 \%$ of baseline $(1.2 \mathrm{~L} / \mathrm{min}$ ) (Figure 4, right), largely due to reductions in tidal volume $(\mathrm{TV}=80 \mathrm{ml}, \mathrm{RR}=15)$. This potentially dangerous respiratory compromise was not noted clinically and no airway maneuvers or other interventions were implemented to address it.

\subsection{Propofol Effects}

Patients received varying doses of propofol and other sedatives for their respective procedures. Fourteen of the

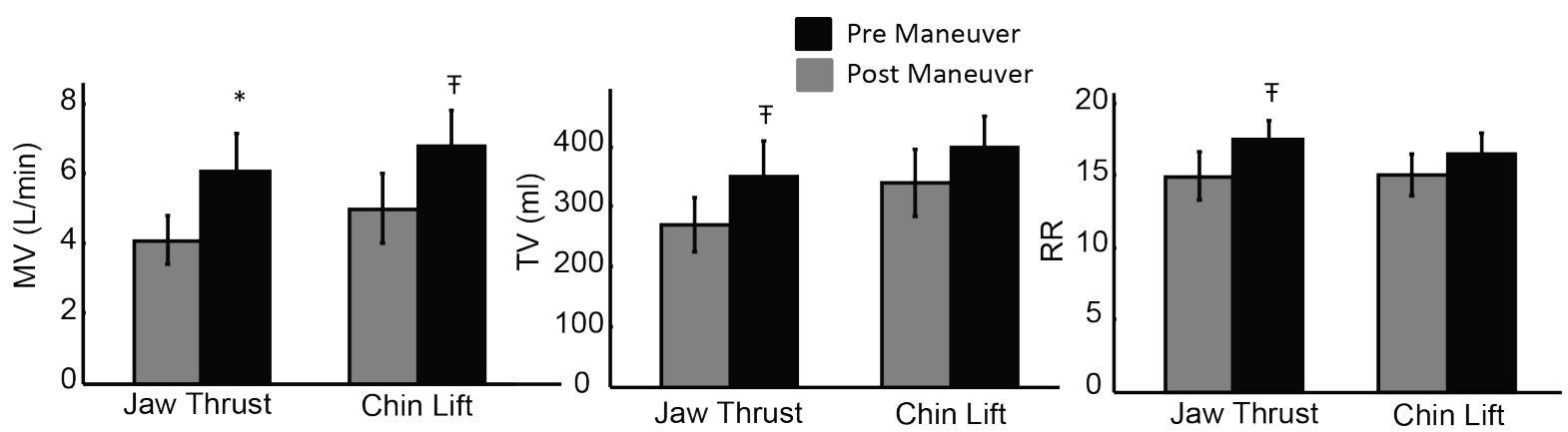

Figure 3. Summary of the Effectiveness of Airway Maneuvers: Minute ventilation (MV), tidal volume (TV) and respiratory rate (RR) pre (gray) and post (black) jaw thrusts and chin lifts in patients undergoing upper endoscopy. Jaw thrusts significantly improved MV from 4.1 to $6.1 \mathrm{~L} / \mathrm{min}$ ( ${ }^{*} \mathrm{p}<0.01$, paired t-test)—an increase of $25 \% \pm 6 \%$. Jaw thrusts also significantly increased TV from 270 to $350 \mathrm{ml}\left({ }^{\mathrm{T}} \mathrm{p}<0.05\right.$, paired t-test), an increase of $16 \pm 5 \%$, and increased RR from $15 \pm 2$ to $18 \pm 1$ $\left({ }^{\mathrm{T}} \mathrm{p}<0.05\right.$, paired t-test) — an increase of $20 \% \pm 8 \%$.Similar to jaw thrusts, chin lifts also significantly improved MV from 5.0 to $6.8 \mathrm{~L} / \mathrm{min}\left({ }^{\mp} \mathrm{p}<0.05\right.$, paired t-test), an increase of $21 \% \pm 9 \%$, but did not have a significant effect on either TV or RR. 

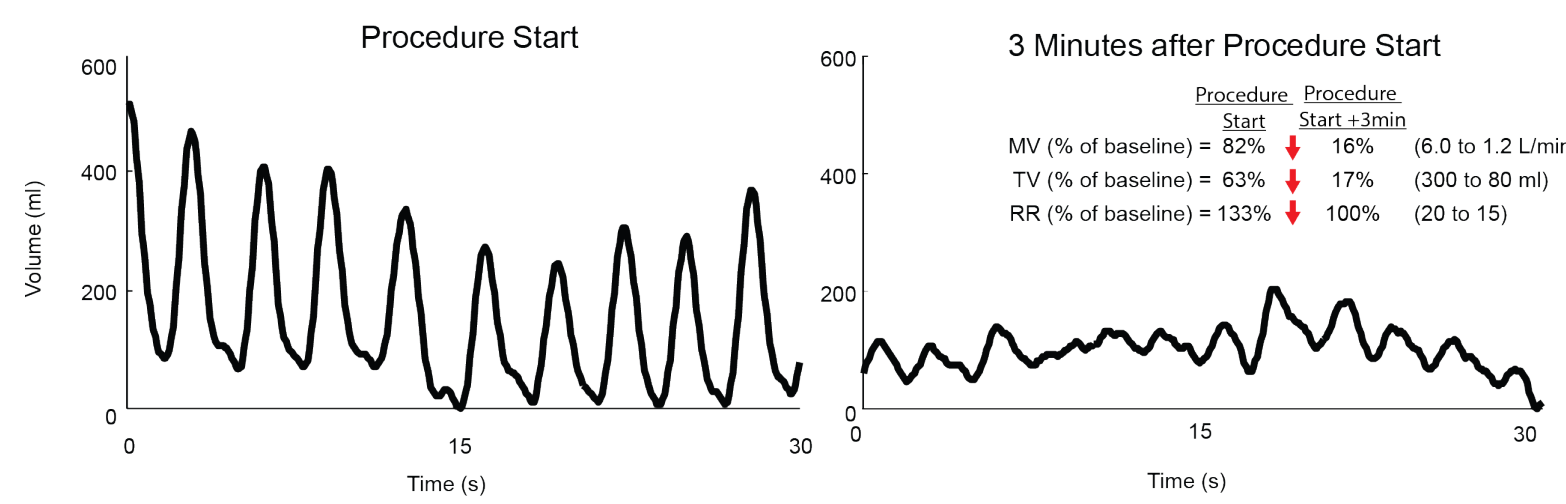

Figure 4. Example of Respiratory Depression Unnoticed by Current Patient Monitoring: Example Respiratory Volume Monitor (RVM) traces and volume measurements from a patient undergoing an esophagogastroduodenoscopy (EGD) where an airway maneuver may have been helpful (healthcare team members blinded to RVM data). Prior to sedative administration or initiation of the procedure, the baseline minute ventilation $\left(\mathrm{MV}_{\text {BASELINE }}\right)$ is $7.3 \mathrm{~L} / \mathrm{min},(16 \%$ above the predicted minute ventilation $\left(\mathrm{MV}_{\mathrm{PRED}}\right)$ of $\left.6.3 \mathrm{~L} / \mathrm{min}\right)$. At the beginning of the procedure, and after sedation, the measured $\mathrm{MV}$ was $82 \%$ of $\mathrm{MV}_{\text {BASELINE }}$ (minute ventilation $(\mathrm{MV})=6.0 \mathrm{~L} / \mathrm{min}$, tidal volume $(\mathrm{TV})=300 \mathrm{ml}$, respiratory rate $(\mathrm{RR})=20$ ). Three minutes into the procedure, after an additional $50 \mathrm{mg}$ bolus of propofol, $\mathrm{MV}$ dropped to $16 \%$ of $\mathrm{MV}_{\mathrm{BASELINE}}$, largely through a reduction in $\mathrm{TV}$ to $17 \%$ of $\mathrm{TV}_{\mathrm{BASELINE}}$, while $\mathrm{RR}$ remains at $100 \%$ of $\mathrm{RR}_{\mathrm{BASELINE}}(\mathrm{MV}=1.2 \mathrm{~L} / \mathrm{min}, \mathrm{TV}=80 \mathrm{ml}, \mathrm{RR}=15)$. Given standard monitoring tools and clinical assessment, the respiratory depression remained unnoticed and no airway maneuver was performed.

25 patients received an infusion of propofol while the other 11 received multiple bolus doses (Table 1 ). At base line, prior to any sedatives, the cohort average $\mathrm{MV}_{\text {BASELINE }}$ was recorded at $9.5 \pm 0.7 \mathrm{~L} / \mathrm{min}(\mathrm{TV}=670 \pm 60 \mathrm{ml}$, $R R=15 \pm 0.7)$. This is higher than the average predicted $M V\left(M_{P R E D}=7.0 \pm 0.2 \mathrm{~L} / \mathrm{min}\right)$. Figure 5 summarizes the recorded trends in MV, TV, and RR as percent of baseline (Figure 5(a)) and as absolute measurements (Figure 5(b)) aligned to the last dose of propofol. One patient had a nearly four-fold increase in MV from baseline until five minutes prior to propofol. This time point coincides with the start of the procedure and is likely due to high patient anxiety prior to the procedure. This patient was removed from the resulting analysis and figure for visual purposes, but significance tests were similar when this patient was included in the analysis. Post-hoc analysis of these trends revealed that MV decreased transiently, reaching a nadir five minutes after the last dose of propofol at $82 \% \pm 10 \%$ of baseline (MV $=7.5 \pm 1.0 \mathrm{~L} / \mathrm{min}$, mean $\pm \mathrm{SEM}$ ). The reduction in MV was driven by a significant reduction in TV (and not RR). At the nadir of MV, the recorded TV was $64 \% \pm 5 \%$ of baseline (TV $=420 \pm 40 \mathrm{ml}, \mathrm{p}<0.001$ w.r.t. baseline) and $17 \% \pm 9 \%$ lower than pre-propofol TV $\left({ }^{\mathrm{F}} \mathrm{p}<0.05\right.$ w.r.t. pre-propofol levels). The RR at the nadir of MV was significantly increased to $123 \% \pm 9 \%$ of baseline ( $R R=17 \pm 0.8,{ }^{\mathrm{F}} \mathrm{p}<0.05$ w.r.t. baseline), indicating that, once again, $\mathrm{RR}$ alone may be a poor indicator of a patient's respiratory status. The effect of propofol on respiration was observed in both continuous infusion and bolus administration protocols.

\section{Discussion}

These results demonstrate that the RVM was able to monitor changes in ventilation before, during and after upper endoscopic procedures, and that it was able to quantify respiratory changes resulting from the administration of medications and clinical interventions, such as airway maneuvers to improve ventilation. Analysis of the respiratory patterns following airway maneuvers showed an increase in MV, TV, and RR.

All patients had a significant reduction in ventilatory volumes after propofol administration. Five minutes after the last dose of propofol, MV and TV were reduced while RR was not, suggesting that monitoring respiratory rate alone is not only insufficient in quantifying the extent of respiratory depression, and can be misleading. Across the cohort, RR increased slightly after the last dose of propofol, likely in an attempt to compensate to the greatly reduced TV.

It is imperative to ensure adequate ventilation during monitored anesthesia care (MAC) at levels of moderate to deep sedation. After the elimination of claims for dental injury, Metzner and Domino's [13] review of the ASA Closed Claim database showed that MAC was used in claims from remote locations (non-operating room (NOR) settings) much more often than in the OR (50\% vs. $6 \%$ ), and that of remote anesthesia locations, $32 \%$ of claims 


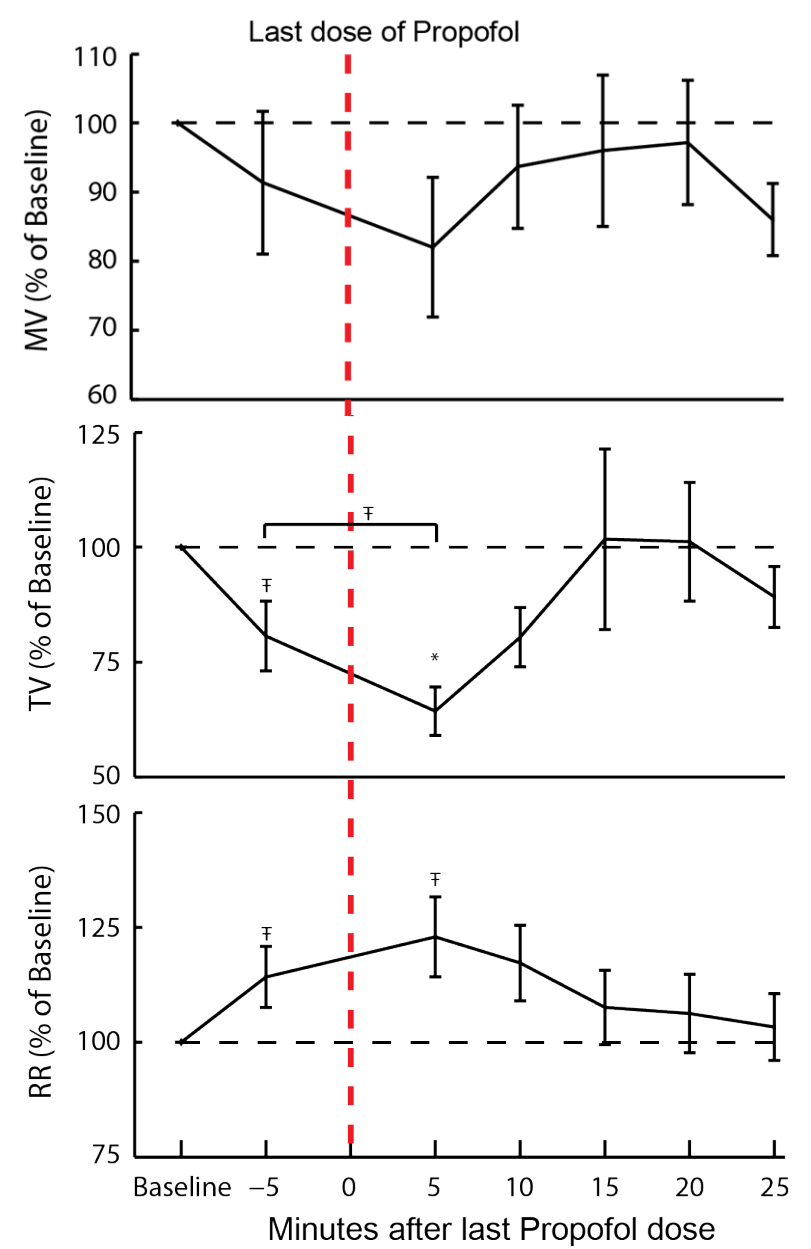

(a)

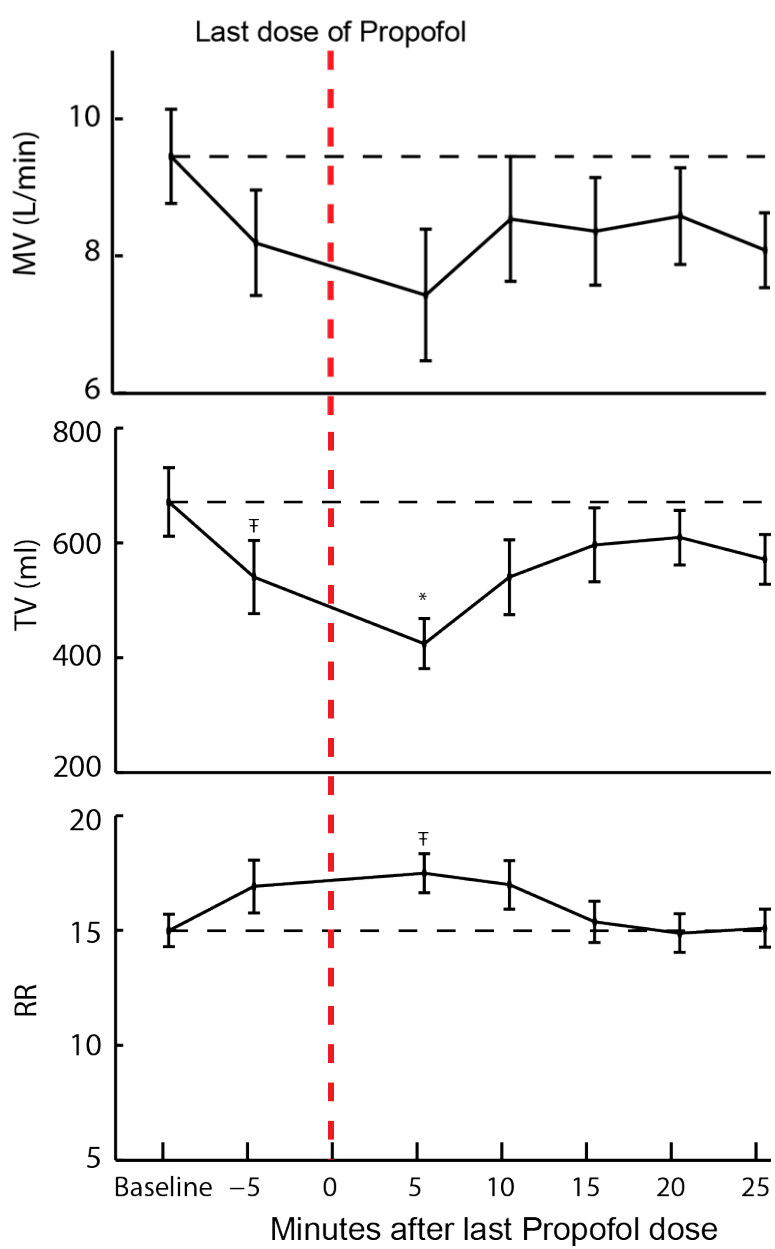

(b)

Figure 5. Respiratory Parameter Trends: (a) Minute ventilation (MV), tidal volume (TV) and respiratory rate (RR) relative to baseline (black dashed line) as measured with a Respiratory Volume Monitor (RVM) before and after the last dose of propofol (red dashed line). Five minutes before the last dose of propofol, MV was $91 \% \pm 10 \%$ of baseline, TV was significantly reduced to $81 \% \pm 8 \%$ of baseline $\left({ }^{\mathrm{F}} \mathrm{p}<0.05\right)$, and RR was significantly increased to $114 \% \pm 7 \%$ of baseline $\left({ }^{\mathrm{T}} \mathrm{p}<\right.$ $0.05)$. Five minutes after the last dose of propofol, MV was $82 \pm 10 \%$ of baseline, while TV was reduced further to $64 \% \pm$ $5 \%$ of baseline $\left({ }^{*} \mathrm{p}<0.001\right)$ and RR was significantly increased to $123 \% \pm 9 \%$ of baseline $\left({ }^{\mathrm{T}} \mathrm{p}<0.05\right)$. TV was also significantly lower 5 minutes after propofol relative to 5 minutes before propofol ( ${ }^{\mathrm{T}} \mathrm{p}<0.05$ ); (b) Absolute values of MV, TV, and $\mathrm{RR}$ relative to the last dose of propofol (red dashed line). At baseline (black dashed line) $\mathrm{MV}=9.5 \pm 0.7 \mathrm{~L} / \mathrm{min}, \mathrm{TV}=670 \pm$ $60 \mathrm{ml}$, and $\mathrm{RR}=15 \pm 0.7$. Five minutes before the last dose of propofol, TV was significantly reduced from baseline to 540 $\pm 60 \mathrm{ml}\left({ }_{\mathrm{F}}^{\mathrm{p}}<0.05\right)$. Five minutes after the last dose of propofol, MV was $7.5 \pm 1.0 \mathrm{~L} / \mathrm{min}$, TV was significantly reduced from baseline to $420 \pm 40 \mathrm{ml}\left({ }^{*} \mathrm{p}<0.001\right)$ and RR was significantly increased from baseline to $17 \pm 0.8\left({ }^{\mp} \mathrm{p}<0.05\right)$.

occurred from cases taking place in the GI suite. Remote locations also had a greater severity of injury; with mortality rate double that of operating room claims. Complications related to inadequate oxygenation/ventilation occurred seven times more frequently in remote locations than in the OR [13]. Under consensus expert opinion, the ASA recommends that monitoring of ventilation be used in all anesthetics achieving moderate to deep sedation [14].

This study suggests that respiratory volume monitoring could optimize ventilation monitoring and direct airway maneuvers during sedation. In the GI suite, space is often limited, patients are usually in a non-supine position, and the airway is shared by the endoscope. This combination can make it more difficult to monitor airway patency and adequate ventilation, and often limits the airway maneuvers available for rescue without pausing or aborting the procedure. The data presented here shows that even in the hands of skilled anesthesiologists, patients can experience inadequate ventilation. Despite existing monitoring techniques and one-on-one clinical 
observation, episodes of clear hypoventilation were not appreciated or addressed. In this sample no adverse outcomes were observed but the ASA Closed Claims data indicates that improvement in the ability to monitor and care for ventilation during sedation procedures is needed. This study shows that the RVM is sensitive to changes in ventilation during upper GI endoscopy. In addition, RVM provides real-time feedback about the adequacy of airway interventions utilized by the anesthesiologist. Prior to use of the RVM, it was difficult to quantify the effectiveness of various rescue airway maneuvers, but with RVM's continuous display of MV, TV and RR values in non-intubated patients, the changes in ventilation created by a given maneuver can be easily quantified and recorded. Further, real time demonstration of effectiveness of the various airway maneuvers may now be used create safer protocols and relied upon to provide feedback during training of healthcare professionals to optimize their performance.

In addition to showing utility of the RVM, this study reiterates the inherent risk of the medications used in procedural sedation. Propofol-based sedation can result in increased respiratory depression, apnea and decreased TV [5]-[8] and it should only be used in situations in which continuous hemodynamic and ventilatory monitoring is available, and only by practitioners who are able to perform advanced airway maneuvers. This is the first known description of the decrease in tidal volume observed with a non-invasive respiratory volume monitor after a final dose of propofol is given or after an infusion is discontinued. The data analysis shows that this decrease does not directly correlate with timing of the end of the procedure. This finding warrants further investigation.

In this study, capnography readings were available to the anesthesiologist, but often noted to be erratic or absent. Specific analysis of capnography data is underway however, data reviewed demonstrated cases in which capnography alone was not sufficient to prompt the anesthesiologist to perform an airway intervention to correct significant respiratory depression and cases in which airway maneuvers were performed at a time of adequate minute ventilation. Acoustic monitoring has been compared to capnography as a sensitive monitor for respiratory rate [15] [16], however this technology does not quantify tidal volume or minute ventilation and has the potential to "count" obstructed breaths.

This study has several limitations. There was no standardized protocol for the type of anesthetic medications given, or method of delivery. The sample size was small and it was an observational study. One benefit was that since the anesthesia care teams were blinded to the RVM data and the MV, TV and RR measurements were not used to modify or direct patient care, an unbiased view of the patient's respiratory status using traditional monitoring techniques was obtained. Additional studies including the comparison of RVM to capnography are ongoing to further assess the utility of RVM during MAC in non-operating room environments. For this study, the RVM was calibrated to a handheld spirometer prior to the procedure to optimize the accuracy of the measurements with less than an average error of $10 \%$ for MV and TV, as previously demonstrated [10]. In clinical practice, it is anticipated that to simplify implementation, a baseline will be obtained in the position of the procedure and the RVM system will be used to observe the respiratory trace and track percent change from pre-anesthetic baseline, as is demonstrated in Figure 4 and Figure 5.

\section{Conclusion}

The endoscopy suite is not a risk-free environment for anesthesia. Although further research is needed, this study clearly supports previous work that the RVM can be a useful tool for monitoring ventilation in patients undergoing upper endoscopic procedures. By providing the anesthesiologist with continuous real-time quantitative information regarding the effect of anesthetics on the patient's respiratory status, RVM can help determine when an airway maneuver or other intervention is needed, and whether the intervention is successful or further intervention is warranted. Improved ventilation monitoring with RVM may help to reduce serious respiratory events and improve patient safety during managed anesthesia care, procedural and procedural sedation.

\section{Funding}

The study was supported by Respiratory Motion, Inc.

\section{Conflict of Interests}

Drs. Holley, Mathews \& Schapiro report no conflict of interest; Dr. Ladd is employed by Respiratory Motion, 
Inc.; Dr. Campana was employed by Respiratory Motion, Inc. at the time this manuscript was written.

\section{References}

[1] Seeff, L.C., Richards, T.B., Shapiro, J.A., et al. (2004) How Many Endoscopies Are Performed for Colorectal Cancer Screening? Results from CDC’s Survey of Endoscopic Capacity. Gastroenterology, 127, 1670-1677. http://dx.doi.org/10.1053/j.gastro.2004.09.051

[2] American Society for Gastrointestinal Surgery (2002) Guidelines for the Use of Deep Sedation and Anesthesia for GI Endoscopy. Gastrointestinal Endoscopy, 56, 613-617. http://dx.doi.org/10.1016/S0016-5107(02)70104-1

[3] Mackenzie, N. and Grant, I.S. (1987) Propofol for Intravenous Sedation. Anaesthesia, 42, 3-6. http://dx.doi.org/10.1111/j.1365-2044.1987.tb02936.x

[4] Faulx, A.L., Vela, S., Das, A., et al. (2005) The Changing Landscape of Practice Patterns Regarding Unsedated Endoscopy and Propofol Use: A National Web Survey. Gastrointestinal Endoscopy, 62, 9-15. http://dx.doi.org/10.1016/S0016-5107(05)00518-3

[5] Hillman, D.R., Platt, P.R. and Eastwood, P.R. (2003) Upper Airway during Anesthesia. British Journal of Anaesthesia, 91, 31-39. http://dx.doi.org/10.1093/bja/aeg126

[6] Taylor, M.B., Grounds, R.M., Mulrooney, P.D. and Morgan, M. (1986) Ventilatory Effects of Propofol during Induction of Anaesthesia. Anaesthesia, 41, 816-820. http://dx.doi.org/10.1111/j.1365-2044.1986.tb13123.x

[7] Goodman, N.W., Black, A.M.S. and Carter, J.A. (1987) Some Ventilatory Effects of Propofol as a Sole Anaesthetic Agent. British Journal of Anaesthesia, 59, 1497-1503.

[8] Cote, G.A., Hovis, R.M., Ansstas, M.A., et al. (2010) Incidence of Sedation-Related Complications with Propofol Use during Advanced Endoscopic Procedures. Clinical Gasterenterology and Hepatology, 8, 137-142.

[9] Overdyk, F.J., Carter, R., Maddox, R.R., Callura, J., Herrin, A.E., et al. (2007) Continuous Oximetry/Capnometry Monitoring Reveals Frequent Desaturation and Bradypnea during Patient-Controlled Analgesia. Anesthesia \& Analgesia, 105, 412-418. http://dx.doi.org/10.1213/01.ane.0000269489.26048.63

[10] Voscopoulos, C., Brayanov, J., Ladd, D., Lalli, M., Panasyuk, A., et al. (2013) Evaluation of a Novel Non-Invasive Respiration Monitor Providing Continuous Measurement of Minute Ventilation in Ambulatory Subjects in a Variety of Clinical Scenarios. Anesthesia \& Analgesia, 117, 91-100. http://dx.doi.org/10.1213/ANE.0b013e3182918098

[11] Du Bois, D. and Du Bois, E.F. (1916) A Formula to Estimate the Approximate Surface Area if Height and Weight Be Known. Archives of Internal Medicine, 17, 863-871. http://dx.doi.org/10.1001/archinte.1916.00080130010002

[12] American Academy of Respiratory Care Protocol Committee (2003) AARC: Adult Mechanical Ventilator Protocols. http://www.aarc.org/resources/protocol resources/documents/general vent.pdf

[13] Metzner, J. and Domino, K. (2011) Risk of Anesthesia Care in Remote Locations. APSF Newsletter. http://www.apsf.org/newsletters/html/2011/spring/06_remotelocation.htm

[14] American Society of Anesthesiologists (2010) Standards for Basic Anesthetic Monitoring. http://www.asahq.org/For-Healthcare-Professionals/Standards-Guidelines-and-Statements.aspx

[15] Mimoz, O., Bernard, T., Gaucher, A., Fransca, D. and Debaene, B. (2012) Accuracy of Respiratory Rate Monitoring Using a Non-Invasive Acoustic Method after General Anaesthesia. British Journal of Anaesthesia, 108, 872-875. http://dx.doi.org/10.1093/bja/aer510

[16] Ramsay, M., Usman, M., Lagow, E., Mendoza, M., Untalan, E., et al. (2013) The Accuracy, Precision and Reliability of Measuring Ventilatory Rate and Detecting Ventilatory Pause by Rainbow Acoustic Monitoring and Capnometry. Anesthesia and Analgesia, 117, 69-75. http://dx.doi.org/10.1213/ANE.0b013e318290c798 
Scientific Research Publishing (SCIRP) is one of the largest Open Access journal publishers. It is currently publishing more than 200 open access, online, peer-reviewed journals covering a wide range of academic disciplines. SCIRP serves the worldwide academic communities and contributes to the progress and application of science with its publication.

Other selected journals from SCIRP are listed as below. Submit your manuscript to us via either submit@scirp.org or Online Submission Portal.
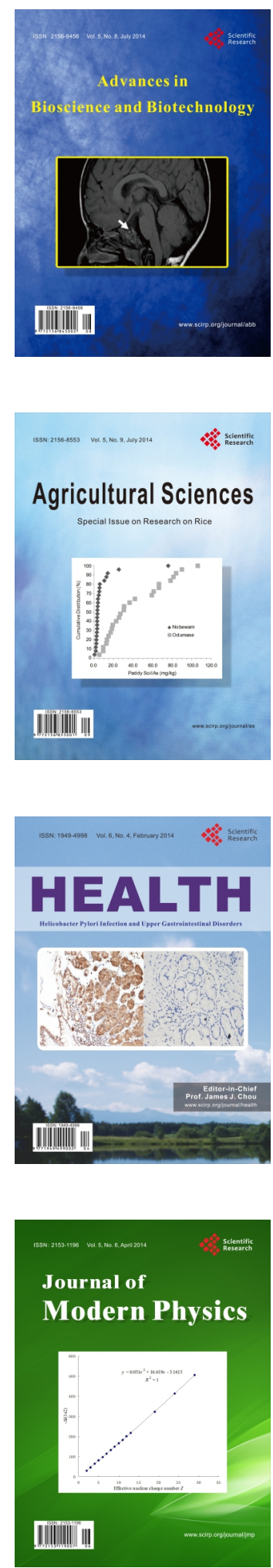
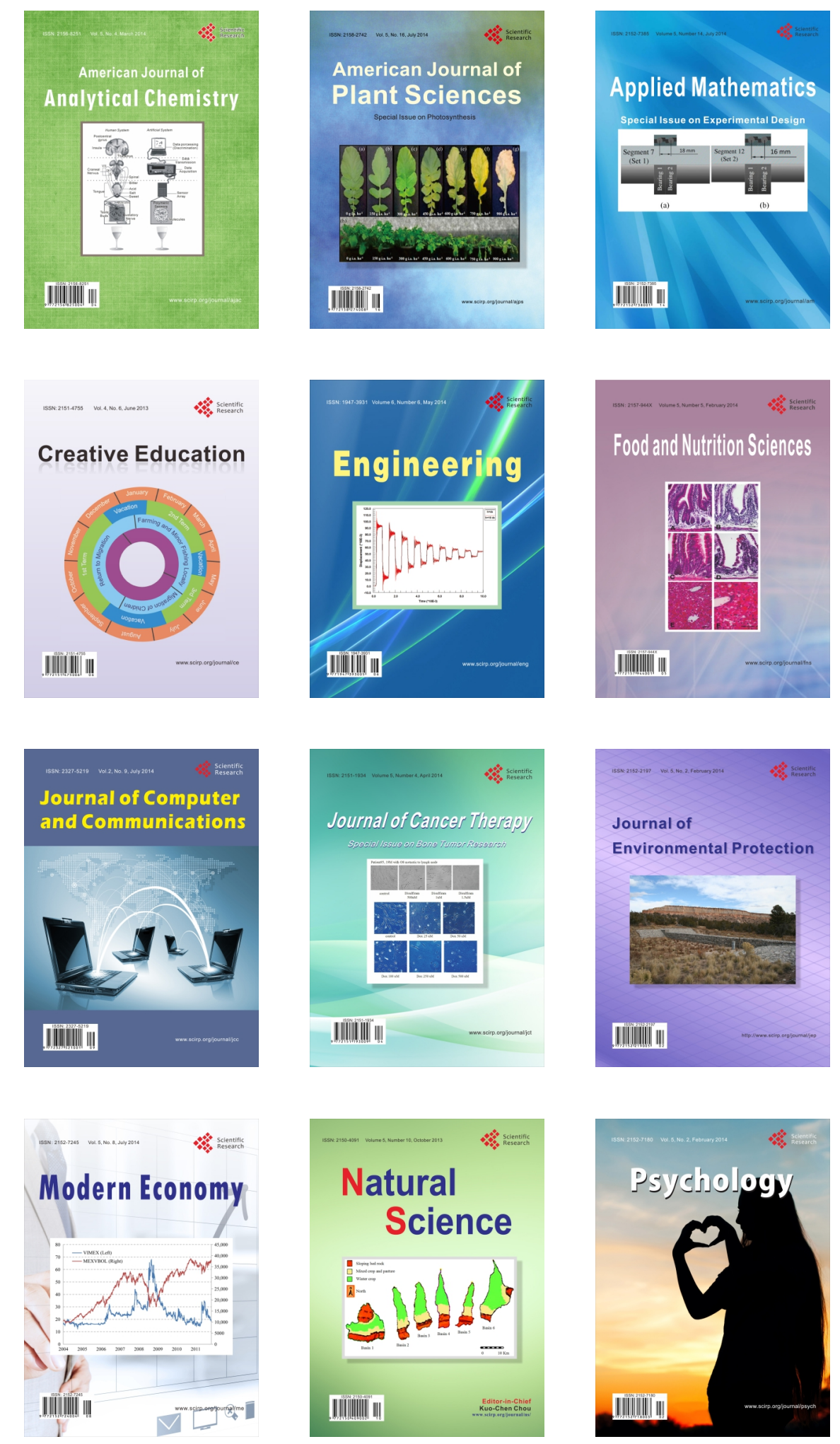